\title{
Técnicas de producción cerámica de Imbabura: una reflexión arqueológica y de saberes locales en la Sierra Norte del Ecuador
}

Techniques de production de céramique à Imbabura : une réflexion sur l'archéologie et les savoirs-faires locaux de la Sierra nord équatorienne Ceramic production techniques in Imbabura: archaeological reflection and local knowledge in the northern highlands of Ecuador

\section{Sthefano Serrano Ayala}

\section{OpenEdition}

\section{Journals}

Edición electrónica

URL: https://journals.openedition.org/bifea/11634

DOI: $10.4000 /$ bifea. 11634

ISSN: 2076-5827

Editor

Institut Français d'Études Andines

Edición impresa

Fecha de publicación: 1 abril 2020

Paginación: 63-84

ISSN: 0303-7495

Referencia electrónica

Sthefano Serrano Ayala, «Técnicas de producción cerámica de Imbabura: una reflexión arqueológica y de saberes locales en la Sierra Norte del Ecuador», Bulletin de l'Institut français d'études andines [En línea], 49 (1) | 2020, Publicado el 08 agosto 2020, consultado el 24 agosto 2021. URL: http:// journals.openedition.org/bifea/11634; DOI: https://doi.org/10.4000/bifea.11634

\section{cc)}

Les contenus du Bulletin de l'Institut français d'études andines sont mis à disposition selon les termes de la licence Creative Commons Attribution - Pas d'Utilisation Commerciale - Pas de Modification 4.0 International. 


\title{
Técnicas de producción cerámica de Imbabura: una reflexión arqueológica y de saberes locales en la Sierra Norte del Ecuador
}

\author{
Sthefano Serrano Ayala*
}

\begin{abstract}
Resumen
Este artículo insiste en la importancia de la etnografía en las investigaciones arqueológicas de la Sierra Norte del Ecuador. La discusión se enfoca en el proceso productivo de la cerámica en dos localidades de alfareras de la provincia de Imbabura. A través del análisis de Difracción de Rayos X (DRX) de arcillas provenientes de las fuentes, cerámica actual y arqueológica, así como del registro de las técnicas de producción, se desarrolla la discusión en el campo de la cerámica arqueológica del país Caranqui. En este sentido, se aprecia una continuidad entre la cerámica arqueológica y actual, la similitud mineralógica entre las fuentes de arcilla y la cerámica de Imbabura, así como una serie de herramientas y contextos etnográficos que son desestimados en el registro arqueológico.
\end{abstract}

Palabras clave: proceso productivo cerámico, alfareras, caranquis, Andes Septentrionales, Ecuador

\section{Techniques de production de céramique à Imbabura : une réflexion sur l'archéologie et les savoirs-faires locaux de la Sierra nord équatorienne}

\section{Résumé}

Cet article insiste sur l'importance de l'ethnographie dans les recherches archéologiques de la Sierra nord équatorienne. La discussion se centre sur le processus de production de céramique dans deux localités de potières de la province d'Imbabura. À partir de l'analyse par diffraction de rayons $X$ (DRX) d'argiles issues de mines, de céramiques actuelles et de céramiques archéologiques, ainsi que d'un registre des techniques de production, nous développons la discussion autour de la céramique archéologique de la zone Caranqui. II en ressort une continuité entre la céramique archéologique

Director del Museo Arqueológico de Perucho y presidente de Sacharxeos, Cia. Lda., Ecuador. E-mail: supremoelder@hotmail.com. 
et actuelle, une similitude minéralogique entre les mines d'argile et la céramique d'Imbabura, ainsi qu'une série d'outils et de contextes ethnographiques peu valorisés en archéologie.

Mots-clés : processus de production céramique, potières, Caranquis, Andes Septentrionales, Équateur

\title{
Ceramic production techniques in Imbabura: archaeological reflection and local knowledge in the northern highlands of Ecuador
}

\begin{abstract}
This article stresses the importance of ethnography in the archaeological investigations of northern Andes of Ecuador. The discussion focuses on the production process of ceramics in two potteryproducing localties in the province of Imbabura. Through the analysis of X-ray Diffraction (XRD) of clays from source áreas as well as contemporary and archaeological ceramics, together with the documentation of production techniques, a discussion of archaeological ceramics from the territory of the Caranqui is presented. In this way, it is shown that there is continuity between archaeological and contemporary ceramics, a mineralogical similarity between clay sources and Imbabura ceramics. There is also a quantity of tools that judging from ethnographic contexts are used in ceramic production but are often ignored in the archaeological record.
\end{abstract}

Keywords: ceramic production process, potters, Caranquis, northern Andes, Ecuador

\section{INTRODUCCIÓN}

Las técnicas actuales de producción cerámica de la Sierra Norte del Ecuador son todavía escasamente utilizadas como fuentes para generar interpretaciones alternas del pasado en la arqueología (véase Minc et al., 2016 como una excepción). Aún más, el factor humano ha sido generalmente minimizado por el registro arqueológico sin que las comunidades puedan aportar elementos significativos para dar respuestas distintas al «sentido común» del arqueólogo. Es así que, en este estudio, se ponen de manifiesto los saberes tradicionales de las alfareras de Imbabura que comprenden los procesos de producción, desde las fuentes de arcilla a través del uso de la geología y la Difracción de Rayos $X$ (DRX) para caracterizarlas hasta la manufactura de vasijas, herramientas y uso del espacio en la secuencia operativa. Finalmente, se presenta una reflexión de los saberes de las alfareras actuales desde la etnoarqueología a fin de generar una discusión en la cerámica arqueológica de la Sierra Norte del Ecuador para el Periodo de Integración Tardío. De este modo, no solo se consigue la articulación de varias ciencias y disciplinas, sino también la posibilidad de que las sociedades y sus saberes, construidos a lo largo de la historia, contribuyan con la producción de conocimiento. 


\section{ETNOARQUEOLOGÍA: EL PAPEL DE LA ETNOGRAFÍA EN LA ARQUEOLOGÍA}

Uno de los aspectos fundamentales que ofrece la etnografía a la arqueología es la posibilidad de obtener datos de procesos productivos actuales que podrían estar relacionados con los del pasado. En el caso de las alfareras actuales de Imbabura, existiría la posibilidad de que los procesos de producción cerámica guarden continuidad con los procesos productivos de la cerámica arqueológica caranqui. En ese sentido, la etnografía obtenida en las localidades de Alabuela y La Rinconada son un buen referente para empezar a reflexionar sobre este tema, y para eso es importante conocer los alcances de la etnoarqueología.

La etnoarqueología comparte experiencias de dos disciplinas: la antropología y la arqueología, en las que se ofrece la posibilidad de conocer personalmente a ese «otro», de hacerle preguntas, de convivir con él, algo que todos los arqueólogos soñamos para poder interpretar el pasado (González Ruibal, 2003: 9). Por ello, es necesario cuestionarse sobre los puntos a favor y en contra de esta disciplina para que el investigador pueda permitirse romper sus esquemas tradicionales.

Desde un inicio, no fue vista con buenos ojos, sobre todo por la creencia de que los datos etnográficos eran el fiel análogo del pasado (Gould, 1980: 29; Wobst, 1978: 306). Otro punto que generó debate es que las sociedades indígenas actuales se ven sometidas por el proceso de globalización, con lo que adquieren nuevos esquemas de desarrollo y comportamientos de la cultura occidental (Burch \& Ellanna, 1994: 3-4; Stahl, 1993: 237), por lo cual, se ha planteado que las sociedades presentes no pueden servir de referentes análogos de las sociedades pasadas. Entre los cuestionamientos, se resalta la incompatibilidad de la temporalidad de las sociedades estudiadas, modelizando sociedades cazadoras del presente con sociedades del Pleistoceno (Wobst, 1978: 307).

No obstante, la etnoarqueología opera bajo los principios de argumentación analógica y, por lo tanto, los dos elementos de la analogía (la fuente y el sujeto) no deben ser iguales, sino que deben tener ciertas condiciones de comparabilidad (Politis, 2005: 9). Esto quiere decir que la etnoarqueología no reside en un punto de igualdad de datos del presente y del pasado, más bien, su tesis atiende a la estructura lógica de argumentar un comportamiento, tecnología o conocimiento entre términos relacionales que dan sentido a un modelo.

Más tarde, las críticas se concentraron en la ética, tanto en el campo de la antropología como en el de la arqueología, ya que se ha propuesto que utilizar grupos vivos para pensar grupos muertos equivale a instrumentalizar al «otro» (González Ruibal, 2003: 12) como un terreno óptimo para apropiarse de conocimientos y tecnologías que ayuden a interpretar el registro arqueológico. Es indudable que la antropología, la arqueología y la etnoarqueología tuvieron un origen colonial y neocolonial, donde puede notarse una «inmoralidad» en la no justificación del uso del presente de una sociedad para interpretar el pasado de otra (Gosden, 1999: 9). No obstante, el reto está innegablemente 
en el quehacer etnoarqueológico: en primer lugar, como una fuente de argumentación en arqueología y, en segundo lugar, como una opción para hacer oír otras voces y deconstruir los relatos de poder tejidos por Occidente (Bhabha, 1994: 94; Said, 1996: 117). La etnoarqueología, más que mostrar a un «otro» exótico, debe mostrar la posibilidad de que otras sociedades existen (González Ruibal, 2003: 13), la emergencia de comprender otros órdenes de pensamiento y otras formas de identidad cultural (Hernando Gonzalo, 1995: 22-24), porque seguir confiando en un «sentido común universal» no es suficiente (Pétrequin \& Pétrequin, 1992: 213).

Con virtudes y críticas, esta disciplina ha logrado posicionarse como uno de los referentes en la interpretación arqueológica. Es así que, en este trabajo, la etnoarqueología juega un papel importante en términos referenciales para la discusión arqueológica en el pasado y de cultura material en el presente, con el fin de visibilizar estructuras alternas de carácter tecnológico. Bajo estas premisas, se ha decidido abordar la tecnología de producción cerámica de las alfareras de Imbabura, entendiendo el proceso desde el presente, aunque también con una implicancia en el registro arqueológico de la cerámica caranqui de la Sierra Norte del Ecuador.

\section{CULTURA MATERIAL CERÁMICA Y ETNOGRAFÍA: PERSPECTIVAS SUDAMERICANAS}

La cerámica es uno de los objetos más comunes que se encuentran en el registro arqueológico y, a partir de esta, los arqueólogos pueden generar interpretaciones del pasado. Muchas veces se definen estilos, composición de pastas y detalladas tipologías. Sin embargo, en todos los aspectos anteriormente citados, el factor humano queda invisible al no existir mayor detalle de los procesos de manufactura, tecnologías, ideologías, innovaciones y un sinnúmero de relaciones sociales que se corresponden con los objetos cerámicos.

Es por esto que arqueólogos, etnoarqueólogos y antropólogos han ido más allá de los atributos visibles que ofrecen los objetos para proponer interpretaciones y discusiones, tomando como referente el dato etnográfico. Por ejemplo, desde esta perspectiva, han abordado la posibilidad de entender la materialidad de la cerámica asociada a las relaciones sociales entre humanos, artefactos, materias primas, entorno, tecnologías, economía, distribución, ideología y simbolismo.

Así, se hacen notar temáticas que no eran tratadas por la arqueología debido a su aparente invisibilidad en el registro arqueológico. El dato etnográfico avizora nuevas formas de interpretar el pasado y el presente. Cremonte (1989) sugiere que la alfarería tradicional en Jujuy, Argentina, responde a aspectos de la ecología, tecnología y cultura que deben ser analizados desde una perspectiva material de la producción, modificación y disposición eventual de las cosas. Por su lado, Sillar (2009: 117), en la línea de ecología cerámica, manifiesta que las tecnologías cerámicas pueden sufrir variaciones de acuerdo a las materias 
primas disponibles en cada estación del año, modificando también el ritmo de las relaciones de trabajo.

En cuanto a la distribución de la cerámica y de las técnicas, la movilidad es un factor importante para explicar este fenómeno que, a veces, es difícil de entender en el registro arqueológico. Arnold (1994: 497-498) evidencia que los alfareros del poblado de Quinua, provincia de Huamanga, se han mudado a Lima e importan materias primas y arcillas de sus comunidades de origen cercanas a Ayacucho para continuar elaborando cerámica para el mercado artesanal. Asimismo, Ramón Joffré (2011) registra a los alfareros golondrinos, quienes emigran hacia otros lugares para producir vasijas. De esta manera, se explica la diversidad y movilidad de estas tecnologías que permiten dotar a los arqueólogos de hipótesis alternas para interpretar el registro arqueológico.

En cuanto a identidades y tecnologías, los estudios de cultura material cerámica han podido establecer paralelismos entre el presente y el pasado, como es el caso del trabajo de Lara (2017) en los Andes surorientales del Ecuador, desde una perspectiva del enfoque tecnológico. También están los estudios de Sjöman (1989; 1992) de las distintas técnicas alfareras del Ecuador.

\section{3. ÁREA DE ESTUDIO}

El estudio se centra en las localidades de La Rinconada y Alabuela, cantones Ibarra y Cotacachi, respectivamente, provincia de Imbabura, Andes septentrionales del Ecuador (fig. 1A). En estas dos localidades, se hicieron investigaciones etnográficas con tres alfareras que se resisten a la pérdida y olvido de esta práctica. En general, las alfareras son adultas, sus edades oscilan entre los 45 y 65 años, y este oficio lo aprendieron de sus madres, de generación en generación. Se escogieron estas dos localidades, ya que son dos de las últimas que aún conservan técnicas tradicionales, pues no han introducido el torno ni hornos eléctricos y pertenecen al grupo de indígenas quichuahablantes de Imbabura. Lamas (1985) y Sjöman (1992) incluyeron estas localidades en su estudio, además de otras que, en la actualidad, han desaparecido (a excepción de San Roque) o han innovado su producción con herramientas modernas y arcillas foráneas. Se realizaron quince visitas a las localidades mencionadas, entre los años 2014 y 2018, tanto a los talleres cerámicos como a las fuentes de arcilla.

La Rinconada se encuentra próxima a la Cordillera Real (Oriental) y Alabuela, cerca de los Andes occidentales de Imbabura. Ambos poblados se localizan en el valle interandino ecuatoriano, a 2600 y 2900 m s. n. m., respectivamente, a $25 \mathrm{~km}$ de distancia el uno del otro. En general, el paisaje de Imbabura ha sufrido grandes cambios generados por la actividad volcánica de ambas cordilleras: hacia el este, por los depósitos volcánicos de Angochagua, asentados sobre formaciones geológicas metamórficas que han modelado los pequeños valles de La Rinconada; hacia el oeste, por los volcanes Imbabura, Cotacachi y Cuicocha que presentan actividad volcánica reciente y que han delineado los valles, ríos, drenajes y montañas de occidente. 
Sthefano Serrano Ayala

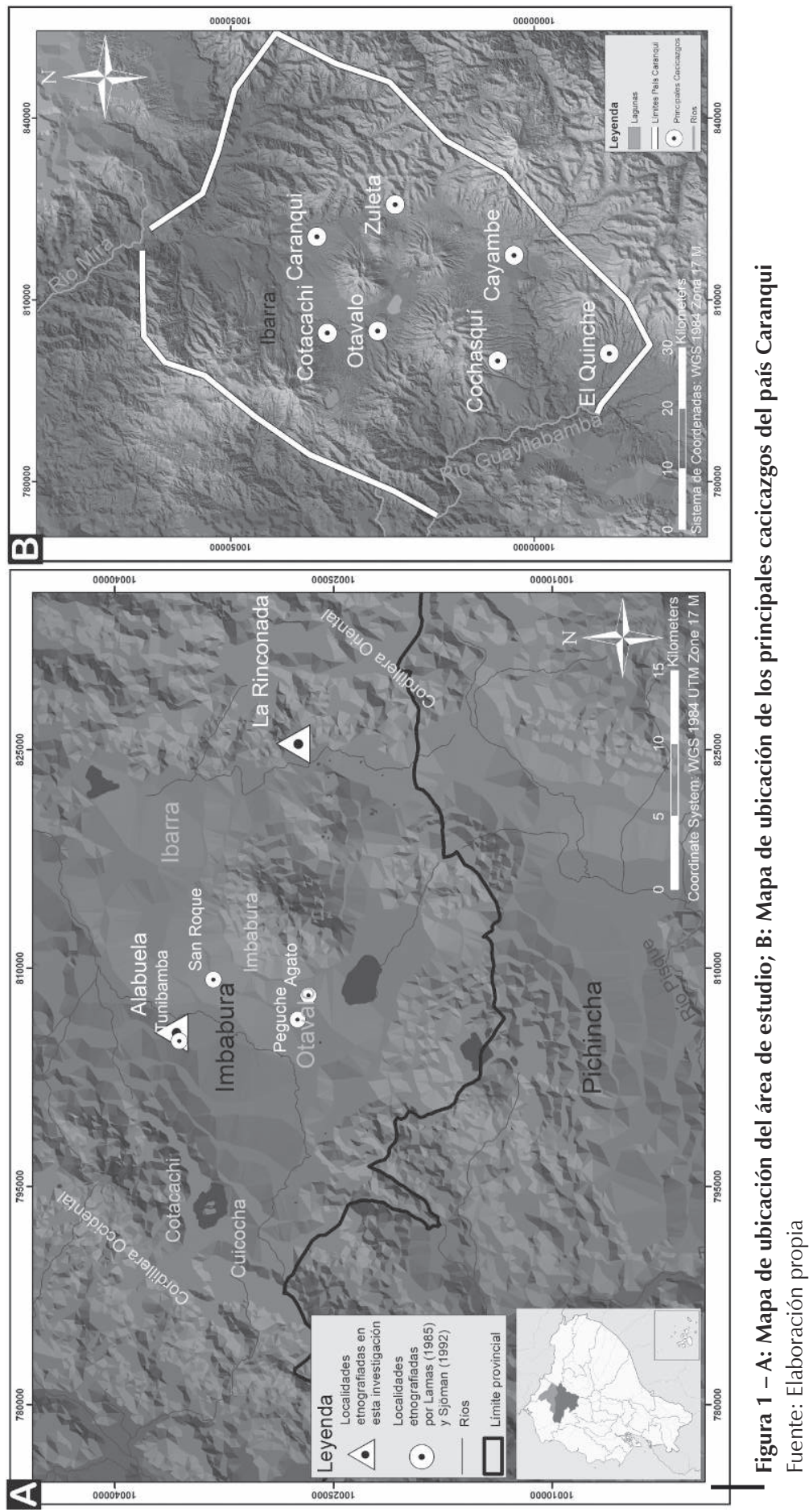




\section{ARQUEOLOGÍA DE LA SIERRA NORTE DEL PERIODO DE INTEGRACIÓN (500-1500 d. C.)}

La arqueología de la Sierra Norte es conocida por su homogeneidad en cuanto a la elaboración de cerámica, principalmente entre las provincias de Pichincha e Imbabura, dentro del Periodo de Integración (500-1500 d. C.). Sin embargo, para el caso en cuestión, se han tomado como referencia las periodizaciones propuestas por Oberem (1981) y Athens (1979), quienes sugieren la división en una etapa temprana que va del 500 al 1250 d. C. y otra tardía que va del 1250 al 1500 d. C.

La etapa temprana se caracteriza por la presencia de montículos hemisféricos con cámaras acampanadas, predomina la cerámica con pintura negativa y, sobre todo, vasijas tipo zapato. Además, se reporta con mayor abundancia la presencia de cerámica cosanga, originaria del este de la Cordillera Oriental y de las selvas altas amazónicas del norte ecuatoriano.

Para la etapa tardía, esta área cultural se destaca por su arquitectura (montículos hemisféricos y pirámides truncas) y formas cerámicas, como ánforas y vasijas trípodes (véase la fig. 5 más adelante). Con base en estos elementos, se sugiere la existencia de la etnia caranqui, un grupo cultural homogéneo y jerarquizado (Espinosa Soriano, 1988), asentado en un territorio conocido como país Caranqui (Espinosa Soriano, 1988; Bray, 2003) que, de acuerdo a la etnohistoria, estuvo conformado por una serie de cacicazgos principales entre el río Mira, al norte (Imbabura); el río Guayllabamba, al sur (Pichincha); y entre las dos cordilleras, al este y oeste (Occidental y Oriental), los cuales son: Caranqui, Cotacachi, Otavalo, Zuleta, Cayambe, Cochasquí y El Quinche (véase la fig. 1B).

Ahora bien, respecto a la cerámica caranqui, existen trabajos con descripciones amplias (Athens, 1979; Echeverría Almeida \& Uribe Alarcón, 1995; Jijón y Caamaño, 1997; Montalvo Puente, 2011; Oberem, 1981; Bray, 1995a). Por lo general, las preguntas de investigación de estos estudios definen, principalmente, tipologías y técnicas decorativas y, muy poco, las tecnologías. Por esto, se cuenta con escasa información detallada de las fuentes de arcilla, talleres, herramientas, técnicas de manufactura $y$, especialmente, agencia humana en el proceso de producción. Es por tal motivo que, en esta investigación, se tiene en cuenta la tecnología cerámica desde la etnoarqueología, para poder contar con más elementos interpretativos para la producción de cerámica arqueológica en la Sierra Norte. En este sentido, las alfareras de Imbabura y, en específico, las de La Rinconada y Alabuela ofrecen una información importante para entender las tecnologías cerámicas del país Caranqui.

\section{PRODUCCIÓN CERÁMICA EN IMBABURA, LA RINCONADA Y ALABUELA}

En este estudio se utilizó la etnografía, mediante registros fotográficos, videos, entrevistas abiertas grabadas en audio y participación activa en el proceso de 
extracción de arcillas y elaboración de vasijas. Además, se tomó como base el trabajo de Parsons (1945), Lamas (1985) y Sjöman (1992), quienes realizaron un estudio etnográfico con alfareras y alfareros de Imbabura. Los trabajos citados describen el proceso técnico desde la perspectiva de un oficio en desaparición. No obstante, no se han tomado estos datos para generar interpretaciones en arqueología que permitan la discusión de estos con los datos de los pueblos precolombinos del país Caranqui; a excepción del trabajo de Minc et al. (2016), quienes, mediante Activación Neutrónica, analizan las fuentes de arcilla utilizadas por alfareros actuales de Imbabura para comparar sus resultados con la cerámica arqueológica caranqui.

En general, el oficio de la alfarería se está perdiendo debido a la poca remuneración económica que genera $y$, principalmente, debido al incremento del uso de recipientes plásticos, metálicos y de porcelana en el diario vivir de la gente de las ciudades y comunidades indígenas. Solo la vasija tipo tiesto o tostador tiene una mejor acogida en los mercados locales y en las ciudades, a veces como elemento decorativo o como utensilio para la preparación de tortillas de maíz. Otras formas, como ollas, jarros, platos y fuentes, se han perdido por la falta de demanda en los mercados. No obstante, dos alfareras de La Rinconada y tres de Alabuela se resisten a perder sus técnicas en la elaboración de ollas y jarros. Teniendo en cuenta esto, se describe a continuación el proceso de elaboración cerámica, desde la provisión de materias primas hasta la quema de las vasijas. El relato unifica el proceso de ambas localidades que están emparentadas étnica y lingüísticamente.

\section{1. Fuente de arcilla}

El estudio de las arcillas y sus fuentes en la cerámica de la Sierra Norte ha tenido aproximaciones de carácter cuantitativo. En este sentido, los químicos han liderado estas investigaciones en el establecimiento de minerales distintivos de la cerámica arqueológica de la Sierra Norte y la cerámica foránea (Cosanga) (Bray, 1995b; Cisneros, 2008; Romero Bastidas \& Molestina, 2010). Por otro lado, los arqueólogos han tomado datos de las ciencias exactas para establecer diferencias mineralógicas (Bray, 2003) y de elementos químicos (Yanchar, 2013; Minc et al., 2016), a fin de proponer intercambios a larga distancia e interacción entre poblados caranqui, a través de la movilización de vasijas locales y foráneas.

Por mi lado, he tratado de analizar todos estos datos desde una perspectiva complementaria entre la etnografía, geología y química, específicamente desde las fuentes de arcilla y su mineralogía, ya que, en los casos citados anteriormente, se analizaba la cerámica arqueológica y fuentes de arcilla desde una perspectiva cuantitativa y de elementos químicos con poco énfasis en el componente geológico y estratigráfico. Además, no se comprendía la formación natural de las arcillas y propiedades que el artesano selecciona. En este sentido, el medioambiente y el conocimiento de este juegan un papel importante en la relación artesano-fuente de arcilla. 
Existe el riesgo, en arqueología, de caer en una representación romántica de una fuente de arcilla, que estaría cercana a un arroyo o en lo espeso de un bosque, y es precisamente por esto que no se han investigado las fuentes, pensando que se encuentran en sitios escondidos o a los que solo tiene acceso el alfarero. Lo cierto es que todas las arcillas de la Sierra Norte se forman a partir de materiales de origen volcánico y son suelos que evolucionan con la meteorización del material parental, ya sea flujos de lava, cenizas volcánicas, depósitos de lahar, etc. Los minerales constitutivos de estas arcillas son: albita, andesina, fayalita, olivino, magnetita, etc., minerales típicos de estos ambientes y que pueden datar desde el Pleistoceno hasta el Holoceno (Instituto de Investigación Geológico Minero del Ecuador, 2017; 1973: 1).

Es por esto que dichas arcillas abundan en toda la región interandina de los Andes septentrionales del Ecuador y se encuentran al alcance de todas las unidades domésticas, sin que exista una exclusividad en el uso de una determinada fuente. Sin embargo, de esta gran cantidad de fuentes, los artesanos han seleccionado las que presentan cualidades óptimas para el trabajo, como los colores, la plasticidad, el tamaño del grano, las inclusiones (desgrasante) y la proximidad a sus talleres (Lamas, 1985; Minc et al., 2016).

En la figura 2, se pueden observar los ambientes de estas arcillas y su formación en horizontes de suelo, denominados según sus características y nomenclatura edafológica (United States Department of Agriculture [USDA], 1999) como A, $A C, A B, B, B T$ y $C$ (figs. $2 A$ y $2 B$ ), que se han desarrollado a partir de cenizas o depósitos de lapilli (material parental de estratos en color gris). La meteorización y los procesos de pedogénesis generan la adherencia del material parental a los distintos horizontes de suelo y, junto con factores ambientales, se van compactando, lavando y disgregando. Por ejemplo, los horizontes $\mathrm{C}$ tienen mayor cantidad de arena, cenizas volcánicas retrabajadas y colores en tonalidades amarillentasclaras, mientras que los horizontes de suelo B y BT son más evolucionados, tienen menor cantidad de arenas, estructuras mucho más arcillosas, texturas tabulares, una plasticidad alta y tonalidades que van desde las gamas del café al amarillo. En cambio, los horizontes $\mathrm{A}$ y los transicionales $\mathrm{AC}$ y $\mathrm{AB}$ tienen mayor materia orgánica, restos de carbón, tonalidades oscuras que van desde el café al negro y se ubican en las partes superiores de los suelos y paleosuelos.

Las alfareras de Alabuela y La Rinconada seleccionan las propiedades de las arcillas según sus necesidades, dependiendo de las formas que desean elaborar, incluso mezclan varias arcillas de diferentes horizontes de suelo hasta obtener la pasta óptima. Por lo general, ellas distinguen cuatro tipos de arcilla: colorada, blanca, negra y roja. Estos colores corresponden a los distintos horizontes de los perfiles estratigráficos de suelo que previamente han sido probados. Cabe recalcar que estas arcillas pueden proceder de distintos suelos, paleosuelos, depósitos y geoformas. Por ejemplo, para elaborar tiestos utilizan arcillas de los horizontes $\mathrm{B}$ (coloradas) y C (blancas) y para ollas y jarros mezclan arcillas de los horizontes $\mathrm{B}, \mathrm{BT}$ (rojas) y $\mathrm{C}$ con arcillas de los horizontes $\mathrm{A}$ y $\mathrm{AB}$ (negras) que tienen mayor contenido de agua, materia orgánica y carbones vegetales (figs. 2A y 2B). Esto 

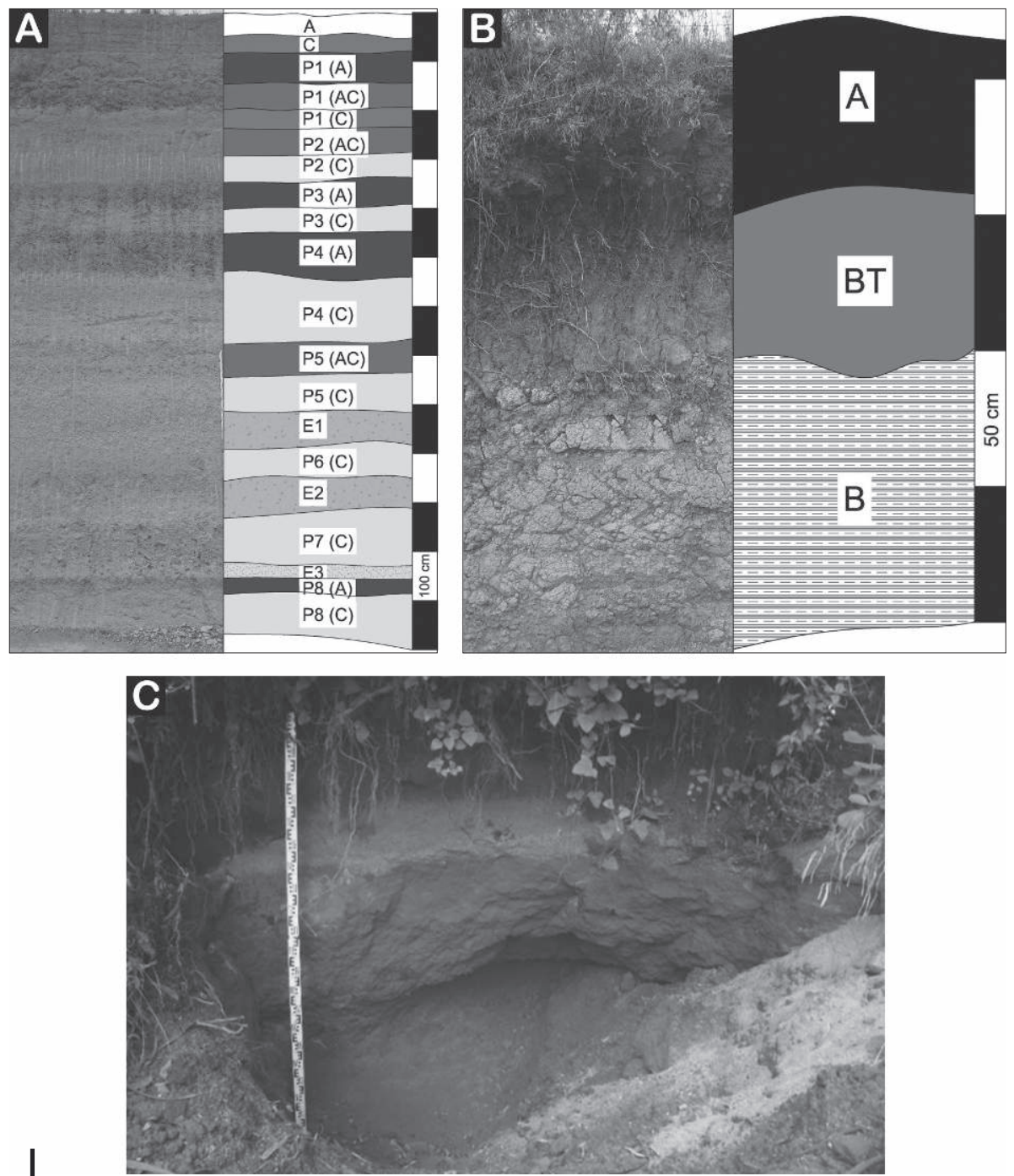

Figura 2 - Horizontes de suelo arcilloso y áreas fuente explotadas en Alabuela: A y B: dibujos de cortes estratigráficos en la región interandina; $C$ : fotografía de una fuente de arcilla en Alabuela

(C) Sthefano Serrano

dependerá de la calidad y diversidad de las arcillas disponibles en cada localidad. En este sentido, las arcillas de La Rinconada suelen ser más plásticas que las de Alabuela.

Para la extracción de las arcillas, en ambas localidades, los hombres y niños hacen cavidades, hoyos y huecos (fig. 2C) con barras, picos y palas. Todo el material se pone en costales y los hombres junto con los niños trasladan la materia prima 
hasta los talleres, que suelen estar a 200-500 m de la fuente. A veces, se suelen usar dos o tres fuentes distintas para elaborar la pasta, ya que las alfareras están en una constante búsqueda de arcillas óptimas. Por lo general, las geoformas preferidas para extraer arcillas en Alabuela son las quebradas, por donde se accede a formaciones geológicas más profundas y antiguas. En cambio, en La Rinconada, se obtienen arcillas del pie de las montañas, en perfiles de suelo menos profundos. Con respecto a la mineralogía, se analizaron por DRX dos muestras de las fuentes de arcilla, una de Alabuela y otra de La Rinconada, que son las pastas principales para realizar todo tipo de vasija. La muestra de Alabuela se obtuvo de un horizonte B sepultado por cenizas volcánicas y la muestra de la Rinconada, de un horizonte B que por erosión aflora en su fuente. En cada caso, se trituró la muestra en un mortero de cerámica independiente y se depositaron las muestras en fundas plásticas para ser enviadas al laboratorio de Metalurgia de la Escuela Politécnica Nacional del Ecuador (Quito).

De los resultados, se obtuvieron minerales distintivos del grupo de las plagioclasas, como albita, andesina, anorita, en su fase cristalina principal, y pirofilita, cordierita, hornblenda, hematita, cuarzo y moscovita, en las fases secundarias de otros minerales (cuadro 1). Los resultados arrojaron datos muy similares a los ya obtenidos por Cisneros (2008) y Romero Bastidas \& Molestina (2010) en sus análisis de DRX para la cerámica arqueológica de valles interandinos de la Sierra Norte.

Cuadro 1 - Resultados cuantitativos de las fuentes de arcilla de Alabuela y La Rinconada

\begin{tabular}{|l|c|c|c|}
\hline \multicolumn{1}{|c|}{ Mineral } & Fórmula & $\begin{array}{c}\text { Alabuela } \\
\text { contenido } \\
\text { (\%) }\end{array}$ & $\begin{array}{c}\text { La Rinconada } \\
\text { contenido } \\
\text { (\%) }\end{array}$ \\
\hline $\begin{array}{l}\text { Plagioclasas } \\
\text { (albita, andesina, anorita) }\end{array}$ & $(\mathrm{Na}, \mathrm{Ca}) \mathrm{Al}(\mathrm{Si}, \mathrm{Al}) \mathrm{Si}_{2} \mathrm{O}_{8}$ & 46 & 47 \\
\hline Pirofilita & $\mathrm{Al}_{2} \mathrm{Si}_{4} \mathrm{O}_{10}(\mathrm{OH})_{2}$ & 15 & 17 \\
\hline Cordierita & $\mathrm{Mg}_{2} \mathrm{Al}_{4} \mathrm{Si}_{5} \mathrm{O}_{18}$ & 13 & 8 \\
\hline Hornblenda & $\left(\mathrm{Ca}, \mathrm{Na}, \mathrm{K}_{2}\left(\mathrm{Mg}, \mathrm{Fe}_{2}, \mathrm{Al}_{5}\right)_{5}(\mathrm{OH}, \mathrm{F})_{2}\left(\mathrm{Si}_{1}, \mathrm{Al}_{2} \mathrm{Si}_{6} \mathrm{O}_{22}\right.\right.$ & 8 & 7 \\
\hline Hematita & $\mathrm{Fe}_{2} \mathrm{O}_{3}$ & 7 & 2 \\
\hline Cuarzo & $\mathrm{SiO}_{2}$ & 6 & 10 \\
\hline Moscovita & $\mathrm{KAl}\left(\mathrm{AlSi}_{3} \mathrm{O}_{10}\right)(\mathrm{OH})_{2}$ & 3 & 7 \\
\hline Hedenbergita & $\mathrm{Ca}\left(\mathrm{Fe}, \mathrm{Mg}_{2}\right) \mathrm{Si}_{2} \mathrm{O}_{6}$ & 2 & - \\
\hline Magnetita & $\mathrm{Fe}_{3} \mathrm{O}_{4}$ & - & 2 \\
\hline
\end{tabular}

Fuente: Elaboración propia 
Una de las diferencias entre ambas arcillas es el porcentaje ligeramente alto de moscovita en las arcillas de La Rinconada, hacia las faldas de la Cordillera Oriental, y menor porcentaje de moscovita en Alabuela, hacia la Cordillera Occidental. La presencia de este mineral de origen metamórfico corresponde a inclusiones pequeñas del material basal de la Cordillera Oriental. Estos minerales sobresalen cuando existen eventos eruptivos de algunos volcanes de esta cordillera. En el caso de los horizontes de suelo cercanos a la Cordillera Real, será común encontrar este mineral en porcentajes más altos. Por otro lado, en Alabuela — que se encuentra hacia la Cordillera Occidental-, la presencia de este mineral responde a la existencia de espesas capas de ceniza de los volcanes del este que depositaron sus piroclastos en el Pleistoceno. Esto es de esperarse, ya que las fuentes de arcilla en Alabuela provienen de quebradas profundas con material volcánico antiguo y, posiblemente, de eventos fuertes de los depósitos volcánicos del Angochagua de la Cordillera Real.

\section{2. Procesamiento de la materia prima}

Luego de obtener las arcillas en ambas localidades, estas son trasladadas en costales hasta los talleres, donde se les disgrega sobre un plástico negro o en esteras de fibras vegetales, según los reportes de Lamas (1985), y se les deja secar al sol durante una semana (fig. 3A). Después, con un pisón (mortero cilíndrico de madera o concreto) o piedras, se tritura la arcilla hasta hacerla polvo. Inmediatamente, se le cierne para quitar minerales excesivamente grandes y se les pone en baldes con agua a fin de decantar los minerales medianos que hayan podido pasar por el cernidor. Más tarde, se saca la arcilla mojada de los baldes y se le prepara adhiriendo otras arcillas (20\%) igualmente trituradas con materia orgánica de horizontes $\mathrm{A}$ y $\mathrm{AB}$, en el caso de La Rinconada, o, simplemente, se trabaja con una pasta única como en Alabuela. Sobre un plástico o cuero de vaca, se pisotea la pasta preparada durante toda una tarde y se le almacena cerca al taller.

\section{3. Elaboración de formas cerámicas}

Las técnicas utilizadas para producir vasijas son similares a las que reportaron Lamas (1985) y Sjöman (1992) para los talleres tradicionales. Además, las técnicas dependen de la forma a elaborar, pudiendo ser a través de moldes, modelado, cordeles o vaciado. En Alabuela y La Rinconada, se toma una porción (1 kg) de la pasta ya preparada y se le comienza a golpear con una mano de piedra sobre otra piedra laja rectangular o también Ilamada mesa o pataquir. Este proceso se repite a partir de las 6 a. m., con el fin de mezclar bien la pasta y quitar posibles burbujas de oxígeno que hayan quedado. 

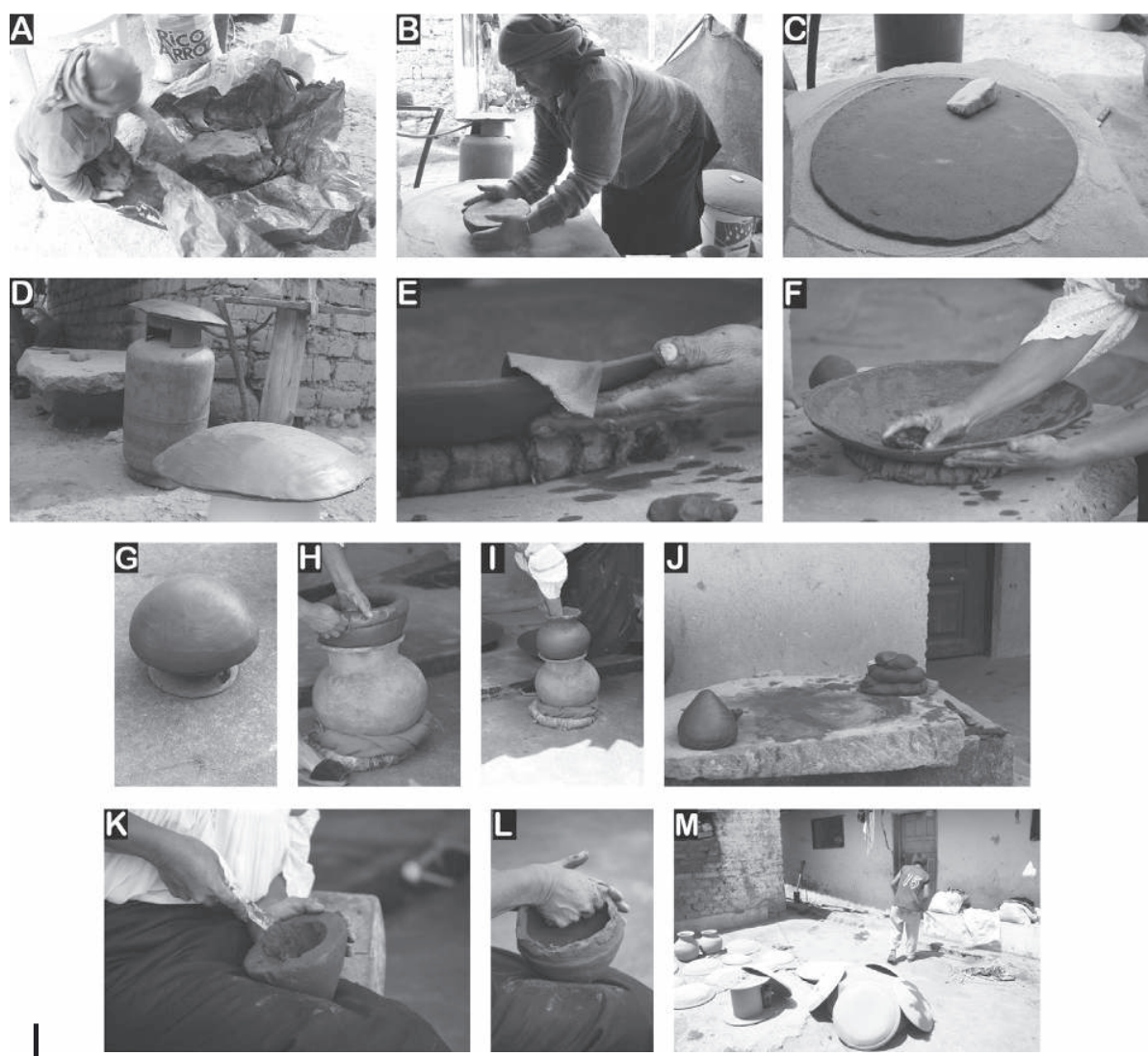

Figura 3 - Proceso de elaboración de formas cerámicas en La Rinconada. Manufactura de tiestos: A: procesamiento de la pasta; B: amasado de la pasta; C: golpeado para la elaboración; D: secado sobre moldes; E: alisamiento con cuero de vaca y giro sobre tazín; F: rotación de tiesto sobre tazín. Manufactura de ollas: G: elaboración sobre molde; $\mathrm{H}$ : uso de cordeles; I: levantamiento de paredes, borde y cuello; J: cono de arcilla para la elaboración de vasijas con base convexa; K: vaciado para manufactura de vasijas; L: vaciado y levantamiento de paredes; M: secado en el patio principal

(c) Sthefano Serrano

\section{3. 1. Molde, modelado y cordeles}

En ambas localidades, luego de tener la pasta óptima, para elaborar los tiestos (comales o tostadores), se forma un cilindro grueso y achatado que es golpeado con una piedra y, al mismo tiempo, se va girando la masa sobre la mesa (fig. 3B). De a pocos, se adhiere polvo de arcilla fino, en el caso de que la pasta se encuentre muy plástica, hasta obtener una circunferencia que es la base para lograr la forma de los tiestos (fig. 3C). Enseguida, se le coloca sobre un tiesto ya quemado que funciona como molde, se le alisa con agua y un fragmento cerámico, para más tarde dejarla secar en la sombra (fig. 3D). 
Después de dos horas, en ambas localidades se moldea mediante el alisado con agua y un borde cerámico y, para acabados más finos, se usa un pedazo de cuero (fig. 3E). Sobre una corona de tela o tazín, que funciona como falso torno, se gira la forma tratando de regularizar las paredes interiores y exteriores del tiesto (fig. 3F). En ocasiones, se suele adherir más polvo de arcilla para cubrir superficies muy mojadas o plásticas y, con un cuchillo, se regularizan los bordes hasta tener lados simétricos. Finalmente, se deja secar una tarde y, al siguiente día, con pulidores de cuarzo y andesita circulares, se alisa el interior y exterior de las paredes, dejándolas secar al sol por una mañana completa. De igual manera, para otras formas, como platos y fuentes, se usa la misma técnica a partir de un molde o de una forma ya elaborada previamente, con el fin de tener recipientes más o menos estandarizados. Esto dependerá de cada unidad doméstica, ya que los tamaños del molde no son similares en las localidades, varían en pocos centímetros el diámetro y el alto de la vasija.

Para formas más elaboradas, como las ollas, el proceso se complementa con cordeles. Es decir, para una vasija globular (fig. 3G), la base está hecha previamente con un molde y las paredes superiores del cuerpo, cuello y bordes son levantados con cordeles. En la figura $3 \mathrm{H}$, se aprecia el molde con la base de una vasija globular previamente trabajada. Luego, con un cuchillo o borde cerámico se hacen incisiones en el borde de esta base, con el fin de que se pegue mejor el cordel. Con $1 \mathrm{~kg}$ de pasta modelada, se realiza un cordel apoyándose sobre la mesa. Más tarde, este se adhiere a la base ya lista y se modela presionándolo con los dedos, a la vez que se alisa el borde cerámico. Poco a poco, se levantan las paredes del cuerpo, y se le deja reposar sobre otra vasija ya quemada. Para el cuello y borde, se obtiene un segundo cordel más pequeño (1 lb), se realizan las mismas incisiones para pegar $y$, con el mismo borde y manos, se logra la forma deseada (fig. 3I). Finalmente, se alisa con un cuero, se deja secar y se pule en seco al siguiente día.

\section{3. 2. Vaciado y cordeles}

En La Rinconada y Alabulela, esta técnica se utiliza para formas con base cónica. En la localidad de Tunibamba, existía una variación: solía utilizarse el cono de la base del penco (Agave americano) para introducir arcilla, para luego ir levantando las paredes de la vasija (Lamas, 1985: 97). Hoy en día, no se utiliza el penco y, más bien, se toman $2 \mathrm{~kg}$ de pasta arcillosa, se da el tratamiento de golpeado sobre la mesa y se modela un cono macizo (fig. 3J). Luego de esto, con un cuchillo se vacía arcilla del interior del cono y se modelan las paredes de la vasija, alisando con un fragmento cerámico (fig. 3K). Finalmente, con dos cordeles (uno grueso para terminar el cuerpo y otro más delgado y pequeño para el cuello y borde) se modela el resto de la vasija (fig. 3L). Se alisa con fragmentos cerámicos y un resto de cuero, se deja secar durante la tarde y la noche a la sombra y, al siguiente día, se alisa con pulidores de piedra y se deja secar al sol (fig. 3M). 


\section{4. Quema}

Para la quema, se utilizan combustibles que pueden ser paja de páramo (Calamagrostis intermedia), ramas, césped y maderas de eucalipto o arbustos de Polylepis, según la disponibilidad de recursos cercanos o lejanos ante la escasez. Esta actividad la realizan las alfareras junto con los hombres de la familia, ya que requiere caminatas y cargas de peso considerables. Luego de esto, en La Rinconada se reúnen todas las vasijas ya terminadas, previamente secadas a la sombra por una semana. Generalmente, un depósito o la habitación de la alfarera sirve como almacén de secado a la sombra. En otros casos, como en Alabuela, se deja secar a la sombra en el patio de visitas de las unidades domésticas.

En ambas localidades, en un segundo patio o patio trasero de la vivienda doméstica asociado a basurales o pequeños huertos, en un espacio con una entrada de aire, se arma el horno. Se van colocando los objetos: primero las vasijas grandes en el centro, luego los tiestos alrededor y las vasijas pequeñas entre los espacios que quedan. En el interior, en los espacios entre las vasijas, se colocan leños grandes y al exterior de la acumulación de vasijas, ramas delgadas. Luego se arma la base del horno con tejas, tiestos quebrados y vasijas rotas; se dejan pequeños canales de acceso de aire en la base y se ponen ramas un poco más gruesas (fig. 4A). Inmediatamente, se prende el horno con un fósforo, iniciando el fuego en el centro, con lo cual rápidamente las llamas se propagan con intensidad. Cada diez minutos, durante una hora, se alimenta el fuego con más ramas hasta que las vasijas estén al rojo vivo. Finalmente, se coloca césped, aserrín o paja de páramo sobre el horno, con el fin de elevar al máximo la temperatura, y se despejan los canales de aire para que el fuego siga vivo (fig. 4B). Se deja de alimentar el fuego y se arroja césped o paja un tanto húmeda para bajar la temperatura paulatinamente; se van cerrando de a poco las cámaras de aire durante media hora. Al siguiente día, después de dejar el horno vivo por una noche completa, con una vara de madera se mueven las ramas carbonizadas para ir sacando progresivamente las vasijas (fig. 4C).
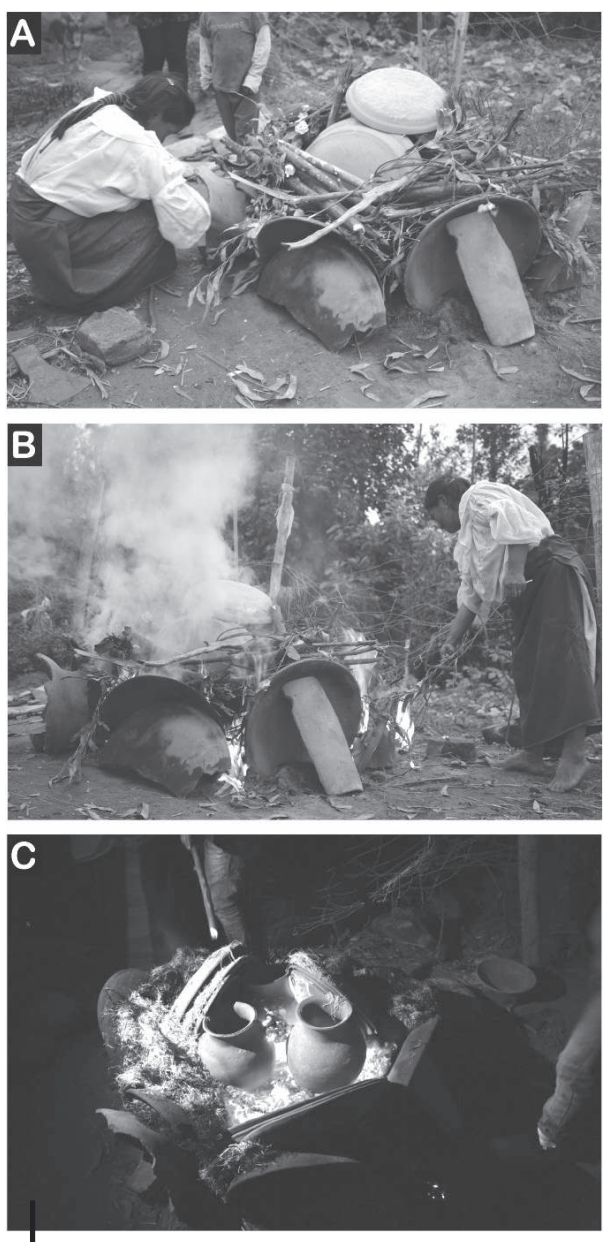

Figura 4 - Proceso de quema en Alabuela: A: armado del horno; B: quema; C: extracción de vasijas cocidas

(c) Sthefano Serrano 


\section{5. Etnoarqueología: una reflexión de saberes del pasado y del presente}

La combinación de resultados etnográficos, geológicos y químicos (arqueométricos) permite generar interpretaciones y reflexiones para la arqueología, principalmente, a través de la formulación de preguntas como la ubicación de las fuentes arcillosas, los procesos de manufactura y la asociación de materias primas arqueológicas de la cerámica Caranqui y la cerámica contemporánea de las alfareras de Imbabura.

En esta investigación, las fuentes de arcilla presentan una mineralogía similar, ya que tienen una formación geológica típica de ambientes volcánicos. Los resultados de DRX de las fuentes de arcilla contienen, en su mayoría, albita y andesina, lo cual se relaciona estrechamente tanto con los análisis de DRX de vasijas elaboradas por las alfareras como con los fragmentos arqueológicos del país Caranqui. Para esto, se analizó un fragmento de un plato de La Rinconada, otro más de un plato de Alabuela y un tercer fragmento de una olla de cerámica arqueológica de La Rinconada en Imbabura. En el cuadro 2, se pueden apreciar los resultados en cuanto a porcentajes de plagioclasas, cuarzo y hornblenda. A nivel mineralógico, son los mismos resultados obtenidos por Cisneros (2008) y Romero Bastidas \& Molestina (2010) en la cerámica arqueológica de la Sierra Norte. Esto sugiere que hay una correspondencia estrecha entre las fuentes de arcilla, cerámica arqueológica y cerámica etnográfica.

En cuanto a la mineralogía, es posible que la cerámica arqueológica y la actual provengan de fuentes similares por la presencia de los minerales ya mencionados. Estos datos concuerdan con la hipótesis de Bray (2003) sobre una producción cerámica a nivel doméstico y de consumo local, esencialmente, por la abundancia de las fuentes y su cercanía a las unidades de vivienda. En este sentido, los horizontes de suelo arcilloso de ambientes volcánicos que están en toda la zona son potenciales fuentes cercanas a los sitios, ya que las formaciones geológicas de origen ígneo en Imbabura y el país Caranqui son homogéneas y se encuentran, principalmente, en quebradas y pie de montañas. En el plano arqueológico, los resultados son similares: a través de análisis elementales de cerámica de distintas formas que abarcan toda la vajilla de los principales sitios arqueológicos del país Caranqui, Yanchar (2013) y Minc et al. (2016) demostraron que la cerámica era elaborada con arcillas cercanas o próximas a los sitios arqueológicos. No se ha podido establecer un grupo químico específico en la cerámica arqueológica que denote especialización y control de la producción cerámica; más bien, las fuentes son espacios abiertos a los pueblos.

Recientemente, los talleres de algunas alfareras han crecido debido al interés de sectores turísticos, que han visto en esta actividad la oportunidad de generar dinero con los saberes de las alfareras. En este sentido, un taller en Alabuela y otro en La Rinconada tienen mayor concurrencia de turistas que compran los objetos y aprenden el largo y duro proceso para la manufactura de vasijas. La producción suele ser un tanto más grande, sin que esto implique que las alfareras desestimen 


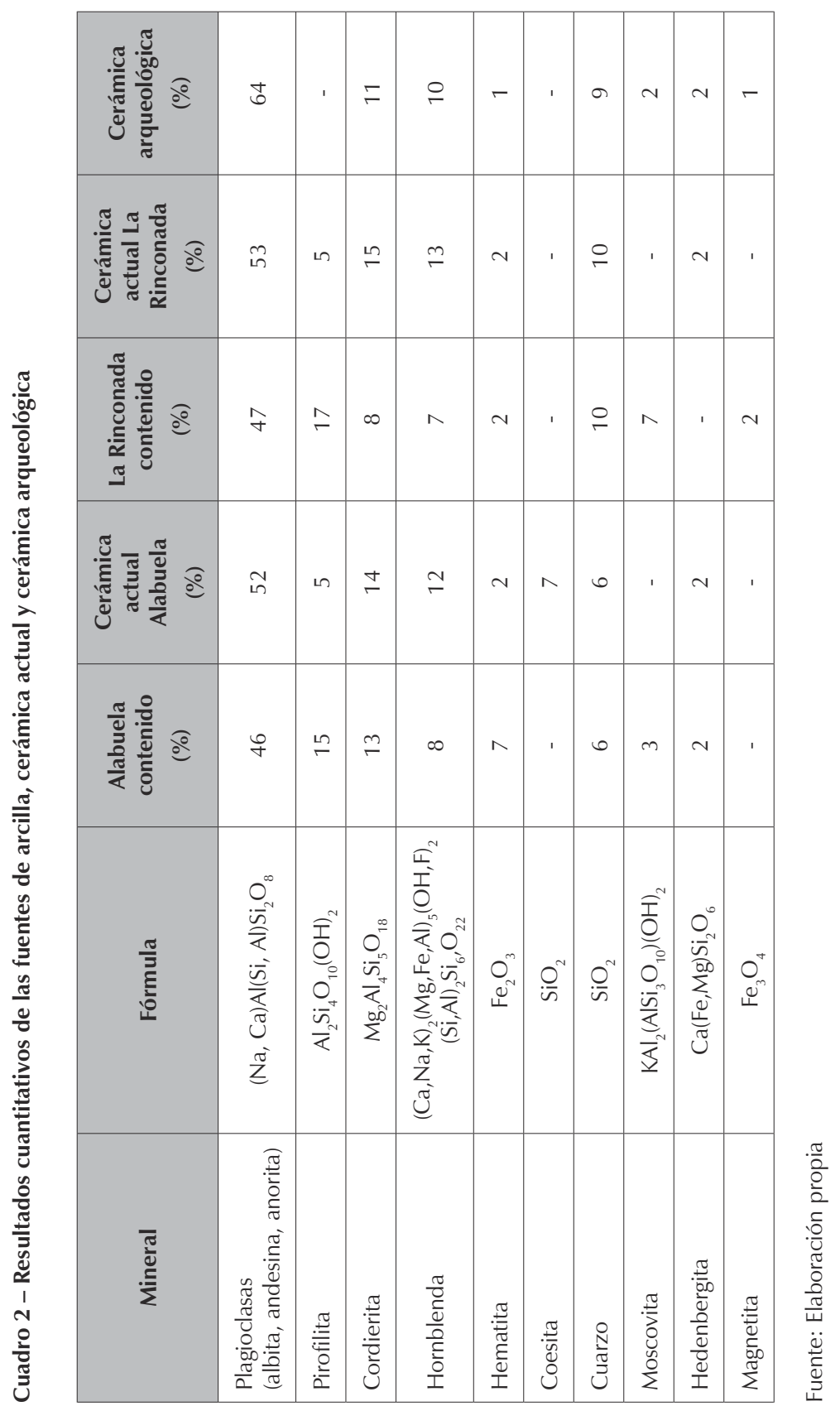


sus otras actividades domésticas. Quizá, esta producción llegue a ser especializada cuando la demanda de turistas sea más amplia en la provincia.

Por otro lado, las herramientas de producción y los espacios del taller obligan a los arqueólogos en Ecuador a repensar los contextos que están excavando. Por ejemplo, las mesas rectangulares de piedra y las manos de piedra pueden estar siendo confundidas con metates o manos de moler. Otros elementos aparentemente descontextualizados, como bordes cerámicos, son fundamentales en el alisado de las vasijas; asimismo, las láminas y lascas de piedra pudieron funcionar en el corte y regularización de formas cerámicas. Herramientas como el falso torno o tazín y alisadores de cuero son imprescindibles; sin embargo, no se conservan en el registro arqueológico. Es así como la etnoarqueología evidencia objetos relevantes en el proceso de manufactura.

En cuanto a los hornos, aparentemente podrían ser confundidos con pisos quemados de viviendas o fogones. No obstante, pueden estar dando pistas sobre la espacialidad de las unidades domésticas y las actividades que se realizan dentro y fuera de una vivienda. El único reporte arqueológico de hornos a cielo abierto, como los que se registran en este trabajo, proviene de las investigaciones de Montalvo Puente \& Dyrdahl (2014) en Ibarra-Imbabura, en un sitio del 800 a. C., donde se observa un rasgo que tiene características similares a las de los hornos de las alfareras de Imbabura. Del mismo modo, los datos mineralógicos de la pirofilita, tanto en cerámica arqueológica actual como de áreas fuente, permiten ahondar en las temperaturas que pudieron alcanzar estos hornos. La pirofilita comienza a disminuir a partir de una temperatura de $700{ }^{\circ} \mathrm{C}$. En la cerámica arqueológica, los porcentajes de pirofilita desaparecen y, en la cerámica actual, disminuyen a más de la mitad del contenido inicial de las fuentes (de 17 a 5 \%) (véase el cuadro 2).

A partir de estos datos etnográficos y arqueológicos, se pueden repensar las estrategias de excavación para dilucidar contextos más claros de las unidades domésticas y sus áreas. En este sentido, la arqueología contextual, a través de la etnoarqueología, puede dotarse de elementos para reconstruir viviendas y espacios exteriores asociados. Es necesario que, como complemento de las perspectivas tipológicas y el establecimiento de cronologías relativas, la cerámica de la Sierra Norte sea mayormente contextualizada a la luz del proceso de producción cerámica y otras actividades que puedan estar asociadas a lo doméstico.

En cuanto a las técnicas, hay una correspondencia importante entre algunas vasijas actuales con formas arqueológicas del país Caranqui, sobre todo del Periodo Tardío. En la figura 5, se puede observar que las formas de la cerámica arqueológica Caranqui y las de la cerámica actual son similares; algunas huellas de manufactura sugieren la aplicación de las mismas técnicas. Por ejemplo, las ollas globulares, tanto arqueológicas como etnográficas, dan cuenta de que la base de la vasija ha sido elaborada a través de un molde y el resto, a través de cordeles. En las vasijas arqueológicas de este tipo es bastante evidente la presencia de huellas de dedos para unir la base y los cordeles que conforman el resto de la vasija. Los platos, fuentes y compoteras se obtienen a partir de moldes 


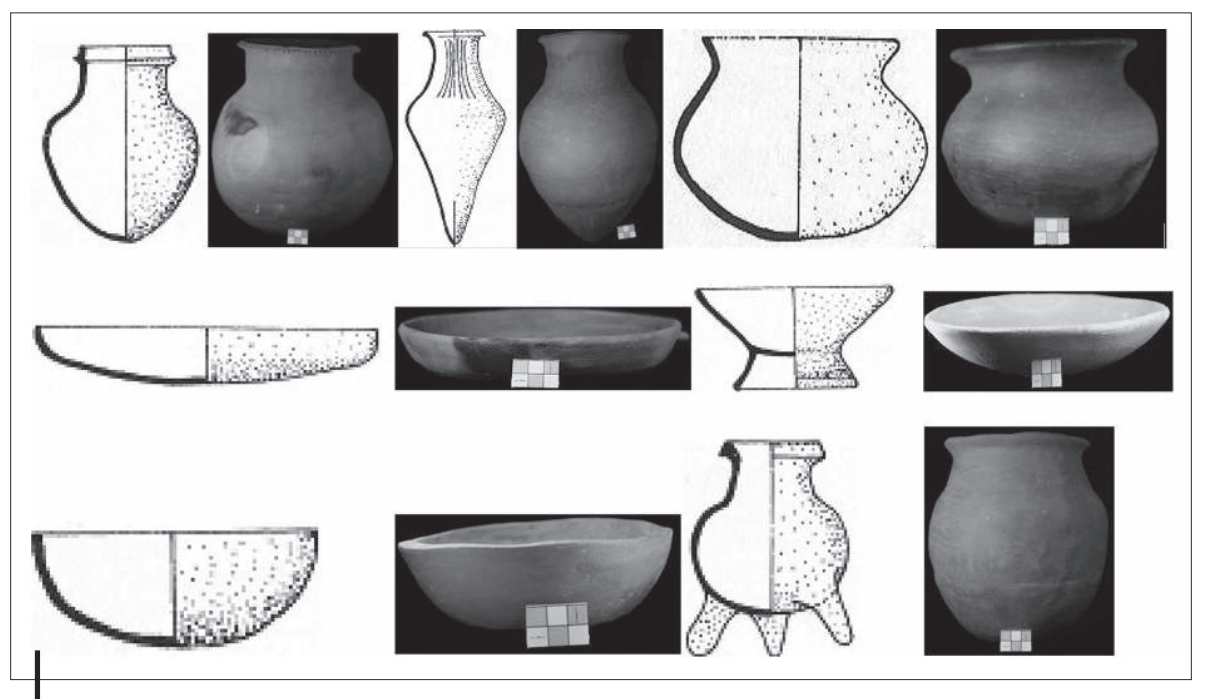

Figura 5 - Dibujos de formas cerámicas del país Caranqui (Meyers en Oberem, 1981) y fotografías de formas cerámicas de manufacturación actual de Alabuela y La Rinconada

(c) Sthefano Serrano

o formas ya hechas, a excepción de los pedestales de las compoteras que son elaborados a partir de cordeles.

Respecto a los jarros grandes con base cónica y ánforas, se puede apreciar el uso de la técnica del vaciado y para el resto del cuerpo el levantado con cordeles. Otras formas típicas del país Caranqui, como las ollas trípode, presentan variaciones de técnicas, como el modelado y cordeles. Dicha forma ha desaparecido en la alfarería actual, pero hay indicios de otras sobre las cuales se pudo trabajar el acoplamiento de los podos, modificando la base y agregando estos elementos.

\section{CONCLUSIONES}

La descripción de las técnicas de producción cerámica de Imbabura da luces para que los arqueólogos puedan reflexionar acerca de la cultura material, la funcionalidad de los objetos, espacios y contextos. De este modo, se da paso a que otras interpretaciones puedan existir en el proceso de investigación arqueológica. Las alfareras de Imbabura y su conocimiento son un testimonio de que la producción cerámica tiene una continuidad desde sus antepasados: los caranquis. Dicho de otro modo, los caranquis aún viven y se resisten a perder una tecnología de larga duración como la cerámica.

Como se aprecia, tanto en la parte arqueológica como etnográfica, existe una relación y una continuidad. Pero también existen cambios profundos como la pérdida de conocimientos por el desuso de objetos, la falta de demanda de los objetos cerámicos o, simplemente, el desinterés por la sabiduría de estas artesanas. 
Sin embargo, estos conocimientos deberían interesar más a los arqueólogos y académicos no solo por la funcionalidad de los datos obtenidos para sus investigaciones, sino porque son conocimientos que nos ayudan a dimensionar otras formas de interpretar el registro arqueológico y la relación entre humanos, objetos y entorno. Se ve la emergencia de incluir y resaltar los conocimientos locales a través de la etnografía y la agencia humana en la arqueología de la Sierra Norte del Ecuador, en complemento de las tipologías cerámicas que, por lo general, han sido la principal fuente para conocer el pasado precolombino.

Por otro lado, en cuanto a las fuentes de arcillas utilizadas hoy en día, podrían estar relacionadas con las del pasado. El análisis geológico y los resultados de DRX de las fuentes de arcilla, vasijas actuales y fragmentos arqueológicos apuntan a un mismo origen por la presencia distintiva de albita y andesina. Esto sugiere un conocimiento pormenorizado de las alfareras respecto a su paisaje natural, las distintas capas de suelo y sus propiedades para ubicar y obtener materias primas para la manufactura cerámica. Bajo esta perspectiva, los análisis cuantitativos se complementan con la información etnográfica que involucra a los agentes humanos en el proceso de extracción de arcillas.

Finalmente, las autoridades gubernamentales e instituciones de patrimonio cultural necesitan emprender esfuerzos para proteger, rescatar y difundir no solo los objetos arqueológicos, sino los agentes de estas producciones que aún viven y se resisten a morir con sus conocimientos.

\section{Referencias citadas}

ARNOLD, D. E., 1994 - Tecnología cerámica andina: una perspectiva etnoarqueológica. In: Tecnología y organización de la producción de cerámica prehispánica en los Andes (I. Shimada, ed.): 477-504; Lima: Fondo Editorial de la Pontificia Universidad Católica del Perú (PUCP).

ATHENS, J. S., 1979 - El proceso evolutivo en las sociedades complejas y la ocupación del Periodo Tardío-Cara en los Andes Septentrionales del Ecuador, 307 pp.; Quito: Instituto Otavaleño de Antropología.

BHABHA, H. K., 1994 - The Location of Culture, xiii + 285 pp.; Londres, Nueva York: Routledge.

BRAY, T. L., 1995a - El conjunto cerámico del País Caranqui: Una interpretación funcional. Marka, 5: 209-235.

BRAY, T. L., 1995b - The Panzaleo Puzzle: Non-Local Pottery in Northern Highland Ecuador. Journal of Field Archaeology, 22 (2): 137-156.

BRAY, T. L., 2003 - Los efectos del imperialismo incaico en la frontera norte: una investigación arqueológica en la Sierra Septentrional del Ecuador, 277 pp.; Quito: Abya Yala.

BURCH, E. S. \& ELLANNA, L. J., 1994 - Introduction. In: Key Issues in Hunter-Gatherer Research (E. S. Bruch Jr. \& L. J. Ellanna, eds.): 1-10; Oxford: Berg Publishers Ltd. 
CISNEROS, P., 2008 - Caracterización del material cerámico en los sitios arqueológicos "La Florida" y "Rumipamba" por las Técnicas de Absorción Atómica y Difracción de Rayos X, 108 pp.; Quito: Universidad Central del Ecuador. Tesis para optar por el título profesional de Químico.

CREMONTE, M. B., 1989 - La alfarería tradicional actual: reflexiones y posibles aplicaciones para la Arqueología a través de dos casos de estudio. Runa, XIX: 117-133.

ECHEVERRÍA ALMEIDA, J. \& URIBE ALARCÓN, M. V., 1995 - Área Septentrional Andina Norte: Arqueología y Etnohistoria, 458 pp.; Quito: Banco Central del Ecuador, Instituto Otavaleño de Antropología, Abya Yala.

ESPINOSA SORIANO, W., 1988 - Los Cayambes y Carangues: Siglos XV-XVI. El Testimonio de la Etnohistoria, 3 tomos; Otavalo: Instituto Otavaleño de Antropología.

GONZÁLEZ RUIBAL, A., 2003 - La experiencia del Otro. Una introducción a la etnoarqueología, 185 pp.; Madrid: Akal.

GOSDEN, C., 1999 - Anthropology and Archaeology. A changing relationship, xiv + 228 pp.; Londres, Nueva York: Routledge.

GOULD, R. A., 1980 - Living Archaeology, xv + 270 pp.; Nueva York: Cambridge University Press.

HERNANDO GONZALO, A., 1995 - La Etnoarqueología hoy: una vía eficaz de aproximación al pasado. Trabajos de Prehistoria, 52 (2): 15-30.

INSTITUTO DE INVESTIGACIÓN GEOLÓGICO MINERO DEL ECUADOR, 1973 - Hoja Geológica Ibarra.

INSTITUTO DE INVESTIGACIÓN GEOLÓGICO MINERO DEL ECUADOR, 2017 - Hoja Geológica Imantag.

JIJÓN Y CAAMAÑO, J., 1997 - Antropología Prehispánica del Ecuador, 387 pp.; Quito: Pontificia Universidad Católica del Ecuador (PUCE), Embajada de España, Agencia Española de Cooperación Internacional, Museo Jacinto Jijón y Caamaño.

LAMAS, V., 1985 - La alfarería tradicional utilitaria en el área de Otavalo y sus inmediaciones. Sarance, 10: 79-126.

LARA, C., 2017 - Aportes del enfoque tecnológico a la arqueología precolombina: pasado y presente de la alfarería en el valle del río Cuyes y su región (Andes sur-orientales del Ecuador), 248 pp.; Oxford: Archaeopress.

MINC, L., YANCHAR, K., BRAY, T. \& ECHEVERRÍA ALMEIDA, J., 2016 - Potting Clays and Ceramic Provenance in Northern Highland Ecuador. In: Vessels Explored: Applying Archaeometry to South American Ceramics and their Production (E. M. Stovel \& G. A. De La Fuente, eds.): 47-63; Oxford: BAR Publishing.

MONTALVO PUENTE, C., 2011 - I materiali ceramici dal sitio Huataviro (Imbabura, Equador): metodo di ricerca; Roma: Universitá degli Studi di Roma-La Sapienza, Facoltá di Filosofia, Lettre, Scienze Umanistiche e Studi Orientali. Tesis de maestría.

MONTALVO PUENTE, C. E. \& DYRDAHL, E., 2014 - Informe Final de las intervenciones realizadas en el yacimiento arqueológico "Las Orquídeas", Parroquia de San Antonio, cantón Ibarra, Provincia de Imbabura, 175 pp.; Quito: Instituto nacional de Patrimonio Cultural (INPC).

OBEREM, U., 1981 - Cochasquí: Estudios Arqueológicos, 3 tomos; Otavalo: Gallocapitán.

PARSONS, E. W. C., 1945 - Peguche, canton of Otavalo, Province of Imbabura, Ecuador: A Study of Andean Indians, viii + 225 pp.; Chicago: University of Chicago Press.

PÉTREQUIN, P. \& PÉTREQUIN, A., 1992 - De l'espace actuel au temps archéologique ou les mythes d'un préhistorien. In: Ethnoarchéologie : justification, problèmes, limites. 
XII Rencontres Internationales d'Archéologie et d'Histoire d'Antibes (F. Audouze): 211-238; Juan-les-Pins: Éd. Association pour la promotion et la diffusion des connaissances archéologiques (APDCA).

POLITIS, G. G., 2005 - Tendencias de la etnoarqueología en América Latina. In: Teoría Arqueológica en América del Sur: 1-44; Olavarría: Instituto de Investigaciones Arqueológicas y Paleontológicas del Cuaternario Pampeano (INCUAPA), Universidad Nacional del Centro de la Provincia de Buenos Aires (UNICEN).

RAMÓN JOFFRÉ, G., 2011 - The Swallow Potters: Seasonally Migratory Styles in the Andes. In: Archaeological Ceramics: A Review of Current Research (S. Scarcella, ed.): 160175; Oxford: Archaeopress.

ROMERO BASTIDAS, M. \& MOLESTINA, M. C., 2010 - Análisis por Difracción de Rayos X de cerámicas de los sitios arqueológicos Rumipamba y La Florida, Quito-Ecuador. In: Las Técnicas Analíticas Nucleares y el Patrimonio Cultural: 73-86; Quito: Instituto Nacional de Patrimonio Cultural (INPC).

SAID, E. W., 1996 - Cultura e imperialismo, 542 pp.; Barcelona: Anagrama.

SILLAR, B., 2009 - La saisonnalité des techniques. Saisonnalité et spécialisation artisanale dans les Andes. Techniques et Culture, 52-53: 90-119.

SJÖMAN, L., 1989 - Las alfareras de Jatunpamba (Cañar), 43 pp.; Cuenca: Fundación Paul Rivet, Museo de Arte Moderno.

SJÖMAN, L., 1992 - Vasijas de Barro: la cerámica popular en el Ecuador, 406 pp.; Cuenca: Centro Interamericano de Artesanías y Artes Populares (CIDAP).

STAHL, A. B., 1993 - Concepts of Time and Approaches to Analogical Reasoning in Historical Perspective. American Antiquity, 58 (2): 235-260.

UNITED STATES DEPARTMENT OF AGRICULTURE (USDA), 1999 - Soil Taxonomy. A Basic System of Soil Classification for Making and Interpreting Soil Surveys, 871 pp.; Washington, D. C.: United States Department of Agriculture, Natural Resources Conservation Service.

WOBST, H., 1978 - The Archaeo-Ethnography of Hunter-Gatherers or the Tyranny of the Ethnographic Record in Archaeology. American Antiquity, 43 (2): 303-309.

YANCHAR, K. C., 2013 - Degree and Scale of Interactions Among Chiefdoms During the Pre-Hispanic Late Period in Northern Highland Ecuador (AD 1250-1525), 289 pp.; Oregon: Oregon State University. 